\title{
Non-Cooperative Regulation Coordination Based on Game Theory for Wind Farm Clusters during Ramping Events
}

\author{
Qi, Yongzhi; Liu, Yutian; Wu, Qiuwei
}

Published in:

Energy

Link to article, DOI:

10.1016/j.energy.2017.05.060

Publication date:

2017

Document Version

Peer reviewed version

Link back to DTU Orbit

Citation (APA):

Qi, Y., Liu, Y., \& Wu, Q. (2017). Non-Cooperative Regulation Coordination Based on Game Theory for Wind Farm Clusters during Ramping Events. Energy, 132, 136-146. https://doi.org/10.1016/j.energy.2017.05.060

- Users may download and print one copy of any publication from the public portal for the purpose of private study or research.

- You may not further distribute the material or use it for any profit-making activity or commercial gain

- You may freely distribute the URL identifying the publication in the public portal 


\title{
Non-Cooperative Regulation Coordination Based on Game Theory for Wind Farm Clusters during Ramping Events
}

\author{
Yongzhi Qi ${ }^{\mathrm{a}}$, Yutian Liu ${ }^{\mathrm{a},{ }^{*}}$, and Qiuwei $\mathrm{Wu} \mathrm{u}^{\mathrm{b}, \mathrm{a}}$ \\ ${ }^{a}$ Key Laboratory of Power System Intelligent Dispatch and Control of Ministry of Education (Shandong \\ University), Jinan 250061, China \\ ${ }^{b}$ Center for Electric Power and Energy, Department of Electrical Engineering, Technical University of \\ Denmark, Kgs. Lyngby, 2800 Denmark
}

\begin{abstract}
With increasing penetration of wind power in power systems, it is important to track scheduled wind power output as much as possible during ramping events to ensure security of the system. In this paper, a non-cooperative coordination strategy based on the game theory is proposed for the regulation of wind farm clusters (WFCs) in order to track scheduled wind power of the WFC during ramping events. In the proposed strategy, a non-cooperative game is formulated and wind farms compete to provide regulation to the WFC during ramping events. A regulation revenue function is proposed to evaluate the competition process of wind farms to provide regulation to the WFC which includes revenue of effective regulation (ER), power support regulation and punishment regulation. The multi-time-interval Nash equilibrium condition is derived for the regulation competition process of wind farms. By setting parameters of the regulation revenue function according to the derived Nash equilibrium condition, the ER strategy is the Nash equilibrium of the regulation competition. Case studies were conducted with the power output data of wind farms from State Grid Jibei Electric Power Company Limited of China to demonstrate the efficacy of the proposed coordination strategy during ramping events.
\end{abstract}

Keywords: Competition process, game theory, Nash equilibrium, ramping events, regulation revenue function, wind farm cluster

\section{Introduction}

Wind power has been developing rapidly over the world for the last few decades. Wind power is variable and uncertain with limited control [1, 2]. It is difficult for wind farms to fully deliver scheduled output due to wind power forecast errors. Large reserve capacities from conventional generation units are required to ensure the security of the system. Especially during ramping events, limited control of wind power increases operating costs and risks of power systems [3-6]. Ramping events are defined as significant changes of wind power output according to a set of rules such as a minimum power swing or duration [4]. In order to improve control performance of wind power, a lot of work has been done on the control of wind turbines, wind farms and wind farm clusters (WFCs) [7-11].

For wind turbines, the focus is maximum power point tracking (MPPT) [7], and frequency and damping control [12-14]. For wind farms, the research aims to improve MPPT and schedule tracking abilities of wind farms. The MPPT of wind farms is to optimize rotor speed and pitch angles of all wind turbines [15]. For schedule tracking, a hierarchical control scheme is usually adopted [16, 17], and it can improve regulation performance of wind farms.

For WFCs, the control is focused on coordination of wind farms. The control optimizes schedules of wind farms and sends active and reactive power control signals to each individual wind farm [9-11]. For the existing wind power coordination methods during ramping events, centralized coordination methods are usually used and the computation is time-consuming. It might be difficult to be deployed in 
real systems for the short-term regulation coordination of wind farms due to the computation burden. Regulation capabilities of wind farms within WFCs may not be sufficiently used to improve the performance of tracking the WFC schedule.

During a ramping event, it is important to track the wind power schedule of the WFC as much as possible and mitigate its impact on the system. In relation to that, an effective coordination strategy is required to improve the tracking performance of WFCs during ramping events. The coordination of wind farms within a WFC requires efficiently distributing the WFC regulation requirement and providing proper revenue to wind farms for the regulation provided.

The game theory has been widely used in power system planning and operation. The game theory is usually employed for cost distribution in order to guarantee a fair outcome [18, 19]. In [20], the game theory was used to distribute the fixed transmission cost of wheeling transactions by a cooperative game according to the transmission usage pattern. The game theory was used in [21] to simulate the decision making process of setting offered prices in a deregulated power system to discourage unfair coalitions. In [22], the game theory was used to model a grid-connected hybrid power system. Furthermore, the existence of the Nash equilibrium was analyzed, and the Nash equilibrium was derived by an iterative algorithm to determine reasonable planning. Reference [23] proposed a method based on the game theory for power system static reserve planning with large-scale wind power to ensure generation capacity adequacy. Reference [24-26] use the game theory to deal with the market behavior of wind power to achieve the equilibrium, which is efficient to cope with uncertainty.

Generally, the game theory is used to deal with the market problems. In the power systems of China and some other countries, there are no day-ahead or real-time electricity markets, especially for wind power coordination. The intention of the work is to develop an efficient incentive scheme to coordinate the production of wind farm clusters during ramping events. In [27], a strategy was proposed to coordinate the charging of autonomous plug-in electric vehicles (PEVs) using the non-cooperative game. At the Nash equilibrium, each PEV reacts optimally with respect to the average of all PEV strategies. In [28], a non-cooperative game was used to deal with costs between the residential energy consumers. A Stackelberg game is established between the utility provider and the energy consumers to realize the demand side management with storage devices. In [29], the game-theoretical model was developed for spinning reserve trading between provincial systems which treats spinning reserve as a commodity. The trading price is calculated by satisfying the Bayesian Nash equilibrium, and the trading quantity is determined.

The research above shows that the game theory is effective to deal with the cost distribution or decision-making to guarantee a fair outcome. The regulation coordination for WFCs is a decision making problem, and the game theory can be used to solve it. Compared to the cooperative game theory, a non-cooperative game is more suitable to distribute the revenue of wind power regulation with uncertainty [30]. The non-cooperative game creates a decentralized framework to coordinate the wind production of WFCs during ramping events. By setting parameters of the revenue functions according to the Nash equilibrium condition, the effective regulation (ER) strategy will be adopted by wind farms when they try to maximize their revenues. With the ER strategy adopted by wind farms, the control error of the WFC is minimized. As such, the revenue maximization of wind farms and the control performance of the WFC are aligned. Therefore, the non-cooperative game based regulation coordination for WFCs during ramping events is a good solution because the computation burden of the optimization is significantly reduced by using the decentralized manner and the control performance is good.

In the proposed strategy, if a ramping event is forecasted to occur, wind farms within a WFC will track both their base schedule and the WFC regulation requirement. The WFC regulation requirement is the difference between the WFC base schedule and the real time power output of the WFC, and will be distributed among wind farms. The wind farms within the WFC compete to provide regulation to the WFC. The regulation of wind farms is determined by the Nash equilibrium of the competition process with the proposed regulation revenue function. In order to guarantee fairness and effectiveness of the competition process, the parameters of the regulation revenue function are set according to the derived Nash equilibrium condition. 
The main contributions of the paper are summarized as follows: (1) propose a non-cooperative coordination strategy based on the game theory to efficiently distribute the WFC regulation requirement during ramping events; (2) define a regulation revenue function for wind farms to evaluate the regulation competition of wind farms; (3) derive the multi-time-interval Nash equilibrium conditions of the wind farms competition.

The paper is organized as follows. Section 2 presents the non-cooperative coordination strategy for the WFC regulation based on the game theory. Section 3 describes the non-cooperative game of the regulation competition within the WFC. The multi-time-interval Nash equilibrium condition of the regulation competition is derived and simplified in Section 4. Case studies are presented and discussed in Section 5 , followed by conclusions.

\section{Coordination Strategy for WFC Regulation}

In this paper, the base schedule of wind farms is determined by the system operator several hours ahead. The WFC base schedule is the sum of the base schedule of wind farms in the WFC. The regulation requirements of wind farms and the WFC vary with control strategies.

Due to the uncertainty and fluctuation of wind power, the maximum available power of wind farms cannot be perfectly forecasted. The base schedule of wind farms and the WFC based on forecasts might not be fully delivered.

In the proposed strategy, the WFC regulation requirement is the difference between the base schedule and real time output of the WFC during ramping events. It is then distributed by a competition process of wind farms in the WFC. The competition process is influenced by the game strategies of wind farms, their regulation capabilities and the economic return. The final regulation from wind farms depends on the game strategy which is analyzed by the non-cooperative game.

The flowchart of the coordination strategy for WFCs during ramping events is shown in Figure 1. The distribution process is divided into two stages and has six steps.

Figure 1 the control flowchart.

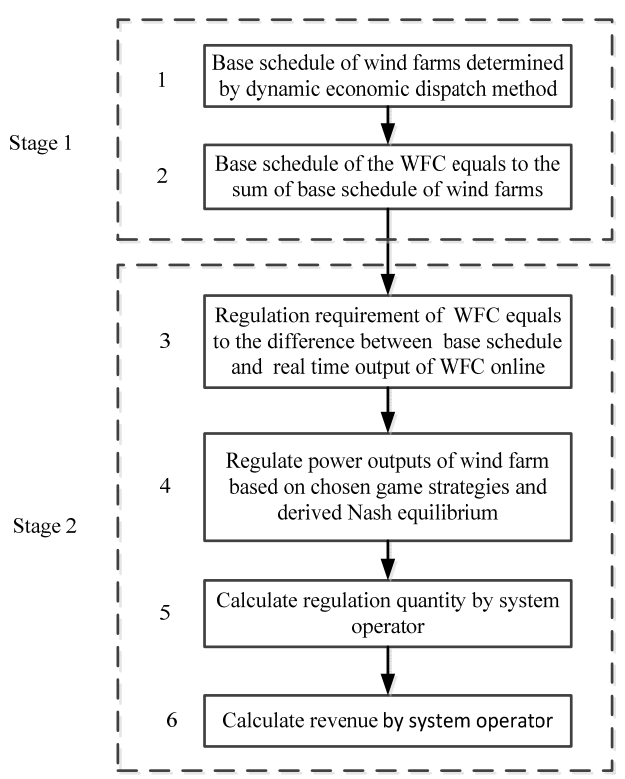

Stage 1 is to determine the base schedule by the system operator several hours ahead.

In Step 1 and Step 2, the base schedules of wind farms and the WFC are determined by the system operator based on a dynamic economic dispatch method.

Stage 2 is the competition process of wind farms to provide regulation to the WFC during ramping events.

In Step 3, when a ramping event is forecasted to occur, the proposed strategy will be activated. The WFC regulation requirement is generated which is the difference between the WFC base schedule and 
WFC real-time power output.

In Step 4, wind farms adjust their power output to provide regulation to the WFC. The regulation from wind farms is influenced by their chosen game strategies and the derived Nash equilibrium. The Nash equilibrium can be derived based on the competition process of the wind farms to distribute the WFC regulation requirement. The followings present the flowchart of the derivation of the Nash equilibrium in Figure 2.

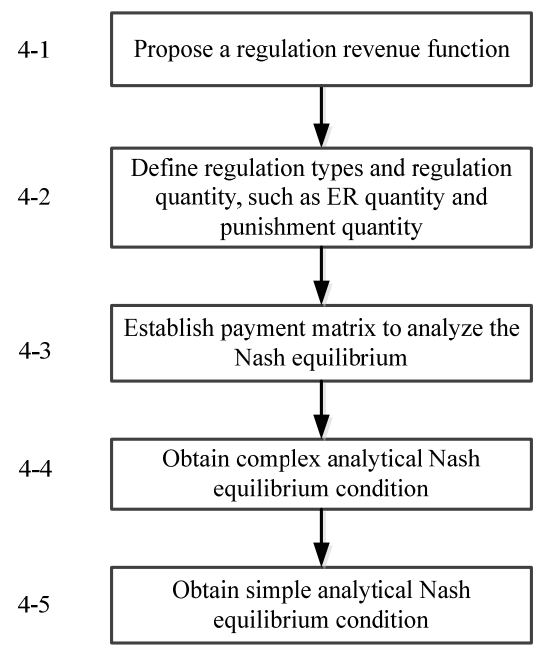

Figure 2 The flowchart of derivation of Nash equilibrium.

In Step 4-1, a regulation revenue function is proposed to evaluate the regulation of the wind farms.

In Step 4-2, regulation types are defined to distinguish different regulation requirements of the WFC in order to decrease the complexity of regulation quantity calculation. Then, the regulation quantity, such as the ER quantity and punishment quantity, is defined to calculate the regulation function of wind farms.

In Step 4-3, the payment matrix is determined to analyze the Nash equilibrium.

In Step 4-4, the complex accurate analytical Nash equilibrium is obtained. However, considering the complex form, it is difficult to set the parameters in the regulation revenue function. Therefore, it is necessary to simplify the Nash equilibrium obtained.

In Step 4-5, the simplified analytical Nash equilibrium is obtained by sufficient condition derivation for complex Nash equilibrium.

In Step 5, the regulation from wind farms is calculated based on their base schedules and actual outputs of wind farms.

In Step 6, the regulation revenue is calculated based on the base schedules of wind farms, actual power outputs and regulation.

\section{Non-Cooperative Game For WFC Regulation Competition}

\subsection{Game Theory}

In Stage 2 of the coordination strategy, wind farms compete to provide regulation to the WFC during ramping events. In the competition process, the objective of wind farms is to maximize the regulation revenue. In the meantime, the WFC regulation requirement needs to be fulfilled. The problem is a decision making process and can be solved by the game theory.

A game usually consists of four key components [22], [30].

1) game player

Game players refer to wind farms in the WFC. The WFC is the supervisor of the game.

2) game strategy 
Wind farms regulate power outputs by different values according to different game strategies. The game strategy $P$ is,

$$
P \in\left[0, \max \left\{P_{\text {map }}-P_{b}, 0\right\}\right]
$$

where $P_{b}$ and $P_{\text {map }}$ are the base schedule and maximum available power of wind farms, respectively.

In order to facilitate deriving the Nash equilibrium in Section 4 , the game strategies are divided into two types, i.e. the ER strategy and the punishment regulation strategy. The punishment regulation strategy has two types, i.e. the over-regulation (OR) strategy during up regulation and the underregulation (UR) strategy during down regulation. Each regulation strategy corresponds to a certain range of power output adjustment which is described in Section 3.3. The game strategy set, $\psi$, is,

$$
\psi=\{E R, U R, O R\}
$$

3) payoff function

The payoff function in this paper refers to the regulation revenue function in (5) in Section 3.2.

4) Nash equilibrium

The Nash equilibrium refers to the strategy set that all the game players believe they choose the optimal strategies under given information and get the maximum revenue according to the strategies of other game players [30]. When the Nash equilibrium exists, all the game players will choose the strategies of the Nash equilibrium to maximize their revenue.

Denote $\left(E_{r 1}, E_{r 2}, \ldots, E_{r j}, \ldots, E_{r n}\right)$ as the payoff set of the game where $E_{r j}$ is the regulation revenue of the $j$ th wind farm. Denote the payoff set $\left(E_{r 1}^{*}, E_{r 2}^{*}, \ldots, E_{r j}^{*}, \ldots, E_{r n}^{*}\right)$ as the Nash equilibrium. If all the wind farms choose $\psi^{*}$, they can achieve the maximum regulation revenue.

\subsection{Revenue Function of Wind Farms}

For wind farms, the operation target is to achieve the maximum revenue. When the revenue can be increased by a certain regulation strategy, this regulation strategy will be adopted by wind farms. When wind farms fulfill the base schedule and provide regulation to the WFC during ramping events, two kinds of revenue can be gained. A revenue function is proposed to calculate the revenue of delivering the base schedule and providing regulation.

The base revenue is expressed as,

$$
E_{b}=\sum_{i=1}^{N} C_{g} \times \min \left\{P_{W F_{-} b}^{i}, P_{W F_{\text {_real }}}^{i}\right\}
$$

where $E_{b}$ is the base revenue, $C_{g}$ is the unit revenue of generation, $P_{W F_{-} b}^{i}$ is the base schedule in the $i$ th time interval, $P_{W F_{-} \text {real }}^{i}$ is real time power output in the ith time interval, $N$ is the total time intervals.

The regulation revenue is,

$$
E_{r}=f\left(C_{r}, \mu, \Delta P_{W F_{-} r}^{i}\right)
$$

where $f\left(C_{r}, \mu, \Delta P_{W F_{-}}^{i}\right)$ is the regulation revenue function, $C_{r}$ is the unit revenue of regulation.

For the regulation revenue, a detailed regulation revenue function is proposed.

$$
\begin{aligned}
& E_{r}=\mu\left(\sum_{i=1}^{N}\left(C_{e} \cdot \Delta P_{W F_{-} e}^{i}\right)+\sum_{i=1}^{N}\left(C_{p s} \cdot P_{W F}^{i}\right)-\sum_{i=1}^{N}\left(C_{p} \cdot \Delta P_{W F_{-}}^{i} p\right)\right) \\
& P_{W F}^{i}=\max \left\{P_{W F_{\text {real }}}^{i}-P_{W F_{-} b}^{i}, 0\right\}
\end{aligned}
$$

where $E_{r}$ is the regulation revenue, $\mu$ is the regulation flag (when real time power output is larger than the base schedule, $\mu$ is set 1 ; otherwise, $\mu$ is set 0$), C_{e}$ is the ER unit revenue, $C_{p s}$ is the power support regulation unit revenue, $C_{p}$ is the punishment regulation unit cost, $\Delta P^{i}{ }_{W_{-} e}$ is the ER quantity in the $i$ th time interval, $P_{W F}^{i}$ is the power support regulation quantity in the ith time interval, $\Delta P_{W F_{-} p}^{i}$ is the punishment regulation quantity in the ith time interval.

Thus the total revenue $E$ is, 


$$
\begin{aligned}
& E=E_{b}+E_{r}=\sum_{i=1}^{N} C_{g} \times \min \left\{P_{W F_{-} b}^{i}, P_{W F_{-} \text {real }}^{i}\right\} \\
& +\mu \cdot\left(\sum_{i=1}^{N}\left(C_{e} \cdot \Delta P_{W F_{-} e}^{i}\right)+\sum_{i=1}^{N}\left(C_{p s} \cdot P_{W F}^{i}\right)-\sum_{i=1}^{N}\left(C_{p} \cdot \Delta P_{W F_{-} p}^{i}\right)\right)
\end{aligned}
$$

\subsection{Regulation Type}

The WFC regulation requirement, $\Delta P_{W F C}^{i}$, is the difference between the WFC base schedule and WFC real time power output.

$$
\Delta P_{W F C}^{i}=P_{W F C_{-} s c h}^{i}-\sum_{j=1}^{N_{w}} P_{W F_{-} j_{-} r e a l}^{i}
$$

where $P_{\text {WFC_sch }}^{i}$ is the WFC base schedule in the ith time interval, $P_{\text {WF__real }}^{i}$ is the real time power output of the $j$ th wind farm in the $i$ th time interval, $N_{w}$ is the total number of wind farms in the WFC.

According to the sign of $\Delta P_{W F C}^{i}$, the regulation is divided into two types: up regulation $\left(\Delta P_{W F C}^{i}>0\right)$ and down regulation $\left(\Delta P_{W F C}^{i} \leq 0\right)$.

The regulation power in the ith time interval, $\Delta P_{W F_{-} r}^{i}$ is defined as,

$$
\Delta P_{W F_{-} r}^{i}=\left\{\begin{array}{c}
P_{W F_{-} \text {real }}^{i}-P_{W F_{-} \text {real }}^{i-1}, P_{W F_{-} \text {real }}^{i}>P_{W F_{-} b}^{i} \\
0 \quad, P_{W F_{-} \text {real }}^{i} \leq P_{W F_{-} b}^{i}
\end{array}\right.
$$

1) Up regulation

During the up regulation, the maximum regulation power allowed, $\Delta P^{i}{ }_{W F_{-}} r_{-}$max , is equal to the WFC regulation requirement.

$$
\Delta P_{W F_{-} r_{-} \max }^{i}=\Delta P_{W F C}^{i}
$$

When the regulation quantity $\Delta P_{W F_{-} r}^{i}$ is less than or equal to $\Delta P_{W F_{-} r_{-} \text {max }}^{i}$, the corresponding regulation is defined as the ER strategy. The ER quantity $\Delta P^{i}{ }_{W F_{-} e}$ and punishment regulation quantity $\Delta P_{W F_{-} p}^{i}$ are,

$$
\begin{aligned}
& \Delta P_{W F_{-} e}^{i}=\max \left\{\Delta P_{W F_{-} r}^{i}, 0\right\} \\
& \Delta P_{W F_{-} p}^{i}=0
\end{aligned}
$$

When $\Delta P^{i}{ }_{W F_{-} r}$ is larger than $\Delta P_{W F_{-} r_{-} \text {max }}^{i}$ the corresponding regulation is defined as the OR strategy. The ER quantity $\Delta P^{i}{ }_{W F_{-} e}$ and punishment regulation quantity $\Delta P^{i}{ }_{W F_{-} p}$ are,

$$
\begin{aligned}
& \Delta P_{W F_{-} e}^{i}=\Delta P_{W F_{-} r_{-} \max }^{i} \\
& \Delta P_{W F_{-} p}^{i}=\Delta P_{W F_{-} r}^{i}-\Delta P_{W F_{-} r_{-} \max }^{i}
\end{aligned}
$$

2) Down regulation

During the down regulation, wind farms are required to reduce their power outputs to meet the WFC regulation requirement. Compared to the up regulation, it is easier to reduce the power outputs of wind farms. Therefore, the regulation quantity of wind farms is set according to the ratio of the average power of a wind farm to the WFC average power. Wind farms will be punished if they do not meet the regulation requirements.

$$
\Delta P_{W F_{-} \text {min_th }_{-}}^{i}=\left|\Delta P_{W F C}^{i}\right| \cdot P_{W F_{-} j}^{i-1} / \sum_{k=1}^{N W} P_{W F_{-} k}^{i-1}
$$

where $\Delta P_{W F_{-} \text {min_th }}^{i}$ is the down regulation minimum threshold in the ith time interval, $P^{i-1} W F_{-} j$ is the average power support regulation quantity of the $j$ th wind farm in time interval $i-1$.

When $-\Delta P_{W F_{-} r}^{i}$ is larger than or equal to $\Delta P_{\text {WF_min_th }}^{i}$, the corresponding regulation is defined as the ER strategy. The ER quantity $\Delta P_{W F_{-} e}^{i}$ and punishment regulation quantity $\Delta P_{W F_{-} p}^{i}$ are,

$$
\begin{aligned}
& \Delta P_{W F_{-} e}^{i}=-\Delta P_{W F_{-} r}^{i}-\Delta P_{W F_{-} \text {min_th }}^{i} \\
& \Delta P_{W F_{-} p}^{i}=0
\end{aligned}
$$

When $-\Delta P^{i}{ }_{W F_{-} r}$ is less than to $\Delta P_{W F_{-} m_{n} t h}^{i}$, the corresponding regulation is defined as the UR strategy. The ER quantity $\Delta P^{i}{ }_{W F_{-} e}$ and punishment regulation quantity $\Delta P_{W F_{-} p}^{i}$ are,

$$
\begin{gathered}
\Delta P_{W F_{-} e}^{i}=0 \\
\Delta P_{W F_{-} p}^{i}=\Delta P_{W F_{-} r}^{i}+\Delta P_{W F_{-} \text {min_t }_{-}}^{i}
\end{gathered}
$$




\section{Nash Equilibrium of WFC Regulation Competition}

\subsection{Derivation of the Nash Equilibrium}

As shown in (3), the base revenue of wind farm is associated with its base schedule and real time power output, and is not affected by other wind farms. The regulation revenue is related to its regulation process and affected by other wind farms. Therefore, the regulation revenue can be analyzed by the game theory.

When the regulation is provided, $\mu$ is 1 and the Nash equilibrium can be derived. When the ER strategy is adopted, the regulation revenue $E_{r_{-} e}$ is,

$$
\begin{aligned}
E_{r_{-} e}= & \sum_{i=1}^{N} C_{e} \cdot \Delta P_{W F_{-} e}^{i}+\sum_{i=1}^{N} C_{p s} \cdot P_{W F}^{i} \\
= & \sum_{i=1}^{N} C_{e} \cdot \Delta P_{W F_{-} e}^{i}+\sum_{i=1}^{N} C_{p s} \cdot\left(P_{W F}^{i-1}+\Delta P_{W F_{-} r}^{i}\right) \\
= & \sum_{i=1}^{N} C_{e} \cdot \Delta P_{W F_{-} e}^{i}+\sum_{i=1}^{N} C_{p s} \cdot P_{W F}^{0}+\sum_{i \in N_{\text {up }}} C_{p s} \cdot(N-i+1) \cdot \Delta P_{W F_{-} \mathrm{e}}^{i} \\
& -\sum_{i \in N_{\text {down }}} C_{p s} \cdot(N-i+1) \cdot \Delta P_{W F_{-} e}^{i}
\end{aligned}
$$

where $N_{u p}$ is the time interval set of up regulation, $N_{\text {down }}$ is the time interval set of down regulation.

When the OR strategy and UR strategy are adopted during up regulation and down regulation, the regulation revenue $E_{r_{-} o_{-} u}$ is,

$$
\begin{aligned}
E_{r_{-} o_{-} u} & =\sum_{i=1}^{N} C_{e} \cdot \Delta P_{W F_{-} e}^{i}+\sum_{i=1}^{N} C_{p s} \cdot P_{W F^{2}}^{i}-\sum_{i=1}^{N} C_{p} \cdot \Delta P_{W F_{-} p}^{i} \\
& =\sum_{i=1}^{N} C_{e} \cdot \Delta P_{W F_{-} e}^{i}+\sum_{i=1}^{N} C_{p s} \cdot\left(P_{W F^{i}}^{i-1}+\Delta P_{W F_{-} r}^{i}\right)-\sum_{i=1}^{N} C_{p} \cdot \Delta P_{W F_{-} p}^{i} \\
= & {\left[\sum_{i \in N_{\text {in }}} C_{e} \cdot \Delta P_{W F_{-} e_{-} \max }^{i}+\sum_{i \in N_{\text {up }}} C_{p s} \cdot(N-i+1) \cdot\left(\Delta P_{W F_{-} e_{-} \max }^{i}+\Delta P_{W F_{-} p}^{i}\right)\right] } \\
& +\left[\sum_{i \in N_{\text {down }}} C_{p s} \cdot(N-i+1) \cdot \Delta P_{W F_{-} r}^{i}\right]-\sum_{i=1}^{N} C_{p} \cdot \Delta P_{W F_{-} p}^{i}+\sum_{i=1}^{N} C_{p s} \cdot P_{W F_{F}}^{0}
\end{aligned}
$$

\begin{tabular}{|c|c|c|}
\hline ER of Player2 & Strategy & OR and UR of Player2 \\
\hline$\left(E_{r_{-} \mathrm{e}}, E_{r_{-} \mathrm{e}}\right)$ & ER of Player1 & $\left(E_{r_{-} e}, E_{r_{-} \text {o } u}\right)$ \\
\hline$\left(E_{\left.r_{\text {o_ou u }}, E_{r_{\mathrm{e}} \mathrm{e}}\right)}\right.$ & OR and UR of Player1 & $\left(E_{r_{0} \text { ou } u}, E_{r_{0} \text { o u }}\right)$ \\
\hline
\end{tabular}

where $\Delta P^{i}{ }_{\text {WF_e_max }}$ is the maximum value of $\Delta P^{i}{ }_{W F_{-} e}$.

Take a two player game as an example to solve the Nash equilibrium [30]. The payment matrix is an effective tool to analyze the Nash equilibrium. Table 1 shows the payment matrix to analyze the Nash equilibrium.

Table 1 Payment matrix.

The first and second items in brackets are the payoffs of game player 1 and player 2, respectively.

Firstly, in order to motivate wind farms to respond to the WFC regulation requirement with the ER strategy, the regulation revenue should be an increasing function to the ER quantity $\Delta P_{W F_{-} e}^{i}$. The larger the ER quantity, the bigger the regulation revenue. The derivative of the regulation revenue to the ER quantity should be strictly positive.

$$
\frac{d E_{r_{-} e}}{d \Delta P_{W F_{-} e}^{i}}=\sum_{i=1}^{N} C_{e}+\sum_{i \in N_{\text {up }}} C_{p s} \cdot(N-i+1)-\sum_{i \in N_{\text {down }}} C_{p s} \cdot(N-i+1)>0
$$

Considering the worst situation, the WFC regulation requirements in all time intervals are negative. In other words, $N_{\text {down }}=\{1,2, \ldots, N\}$. Eq. (22) can be transformed to (23) and (24). 


$$
\begin{gathered}
\sum_{i=1}^{N} C_{e}-\sum_{i=1}^{N} C_{p s} \cdot(N-i+1)>0 \\
C_{e}>\frac{1+N}{2} \cdot C_{p s}
\end{gathered}
$$

When (24) is satisfied, the regulation revenue is an increasing function to the ER quantity $\Delta P_{W F_{-} e}^{i}$. Wind farms will regulate power output to provide larger ER and get larger regulation revenue.

Secondly, if the ER strategy is the Nash equilibrium, larger regulation revenue should be achieved by the ER strategy than the OR strategy or the UR strategy. According to (24), a larger ER quantity corresponds to larger regulation revenue. When all wind farms choose the ER strategy, they will regulate power outputs by $\Delta P^{i}{ }_{W F_{-} e_{-} \max }$ and get the maximum regulation revenue $E_{r_{-} e_{-} \max }$. Therefore, the following inequality holds.

$$
E_{r_{-} e_{-} \max }>E_{r_{-} o_{-} u}
$$

The detailed expression of (25) is shown below.

$$
\begin{aligned}
& \sum_{i=1}^{N} C_{e} \cdot \Delta P_{W F_{-} e_{-} \max }^{i}+\sum_{i=1}^{N} C_{p s} \cdot P_{W F}^{0}+\sum_{i \in N_{\text {No }}} C_{p s} \cdot(N-i+1) \cdot \Delta P_{W F_{-} e_{\text {max }}}^{i} \\
& -\sum_{i \in N_{\text {down }}} C_{p s} \cdot(N-i+1) \cdot \Delta P_{W_{-}-e_{-} \max }^{i}> \\
& {\left[\sum_{i \in N_{\text {up }}} C_{e} \cdot \Delta P_{W F_{-} e_{\text {m }} \max }^{i}+\sum_{i \in N_{\text {Up }}} C_{p s} \cdot(N-i+1) \cdot\left(\Delta P_{W F_{-} e_{\text {m }} \max }^{i}+\Delta P_{W F_{-} p}^{i}\right)\right]} \\
& +\left[\sum_{i \in N_{\text {donm }}} C_{p s} \cdot(N-i+1) \cdot \Delta P_{W F_{-}-}^{i}\right]-\sum_{i=1}^{N} C_{p} \cdot \Delta P_{W F_{-} p}^{i}+\sum_{i=1}^{N} C_{p s} \cdot P_{W F^{\prime}}^{0}
\end{aligned}
$$

When the following inequality holds, $(25)$ is satisfied.

$$
\begin{aligned}
& \sum_{i \in N_{\text {domo }}} C_{e} \cdot \Delta P_{W F_{-} e_{-} \max }^{i}+\sum_{i=1}^{N} C_{p} \cdot \Delta P_{W F_{-} p}^{i}> \\
& {\left[\sum_{i \in N_{N_{p}}} C_{p s} \cdot(N-i+1) \cdot\left(\Delta P_{W F_{-p}}^{i}\right)\right.} \\
& \left.+\sum_{i \in N_{\text {down }}} C_{p s} \cdot(N-i+1) \cdot\left(\Delta P_{W F_{-}}^{i}+\Delta P_{W F_{-} e_{\text {max }}}^{i}\right)\right]
\end{aligned}
$$

In the end, the Nash equilibrium condition is obtained.

$$
\begin{gathered}
C_{e}>\frac{1+N}{2} \cdot C_{p s} \\
\sum_{i \in N_{\text {down }}} C_{e} \cdot \Delta P_{W F_{-}-\text {_max }}^{i}+\sum_{i=1}^{N} C_{p} \cdot \Delta P_{W F_{-} p}^{i}>\sum_{i \in N_{\text {Up }}} C_{p s} \cdot(N-i+1) \cdot\left(\Delta P_{W F_{-p}}^{i}\right) \\
+\sum_{i \in N_{\text {dom }}} C_{p s} \cdot(N-i+1) \cdot\left(\Delta P_{W F_{-} r}^{i}+\Delta P_{W F_{-} \_ \text {_max }}^{i}\right)
\end{gathered}
$$

\subsection{Simplification of the Nash Equilibrium}

Considering the complex form of (29), it is difficult to set $C_{e}, C_{p s}$ and $C_{p}$. Therefore, it is necessary to simplify (29).

Combine (29) with (19), the following equation can be obtained.

$$
\begin{aligned}
& \sum_{i \in N_{\text {dowm }}}\left[C_{e}-C_{p s} \cdot(N-i+1)\right] \cdot \Delta P_{W F_{-} e_{-} \max }^{i} \\
& +\left[C_{p} \cdot N-C_{p s} \cdot \frac{N \cdot(N+1)}{2}\right] \cdot \Delta P_{W F_{-} p}^{i}+\sum_{i \in N_{\text {down }}} C_{p s} \cdot \Delta P_{W F_{-} \text {min_th }}^{i}>0
\end{aligned}
$$

When (31) and (32) hold, (30) is satisfied.

$$
\begin{aligned}
& C_{e}>\frac{1+N}{2} \cdot C_{p s} \\
& C_{p}>\frac{1+N}{2} \cdot C_{p s}
\end{aligned}
$$

Finally, when (31) and (32) hold, the ER strategy becomes the Nash equilibrium. 


\section{Case Study}

\subsection{Case Study Scenarios and Test System}

Three case study scenarios are selected in order to compare the proposed strategy with two existing control modes. The three scenarios are defined as Mode1, Mode2 and Mode3. The case studies are simulated in MATLAB.

In Mode1, wind farms track their own base schedule.

In Mode2, wind farms track the base schedule and WFC regulation requirement. The WFC regulation requirement is distributed equally to wind farms.

In Mode3, it refers to the proposed strategy. Wind farms track the base schedule and WFC regulation requirement. Wind farms compete to provide regulation to the WFC and the distribution is determined by a non-cooperative game.

The test system consists of ten wind farms which are labeled as WF1 WF10. The capacity of each wind farm is $150 \mathrm{MW}$. The regulation speed of wind farms is $10 \% P / \mathrm{min}$, where $P$ is the average power output of wind farms. The maximum available power outputs of wind farms in this paper are based on the data with 1 minute resolution from the State Grid Jibei Electric Power Company Limited of China. WF1 WF3, WF4 WF7 and WF8 WF10 are connected to the external power system at three points of common coupling (PCC). The base schedules of wind farms consist of 8 time intervals of 15 minutes as shown in Figure 3.
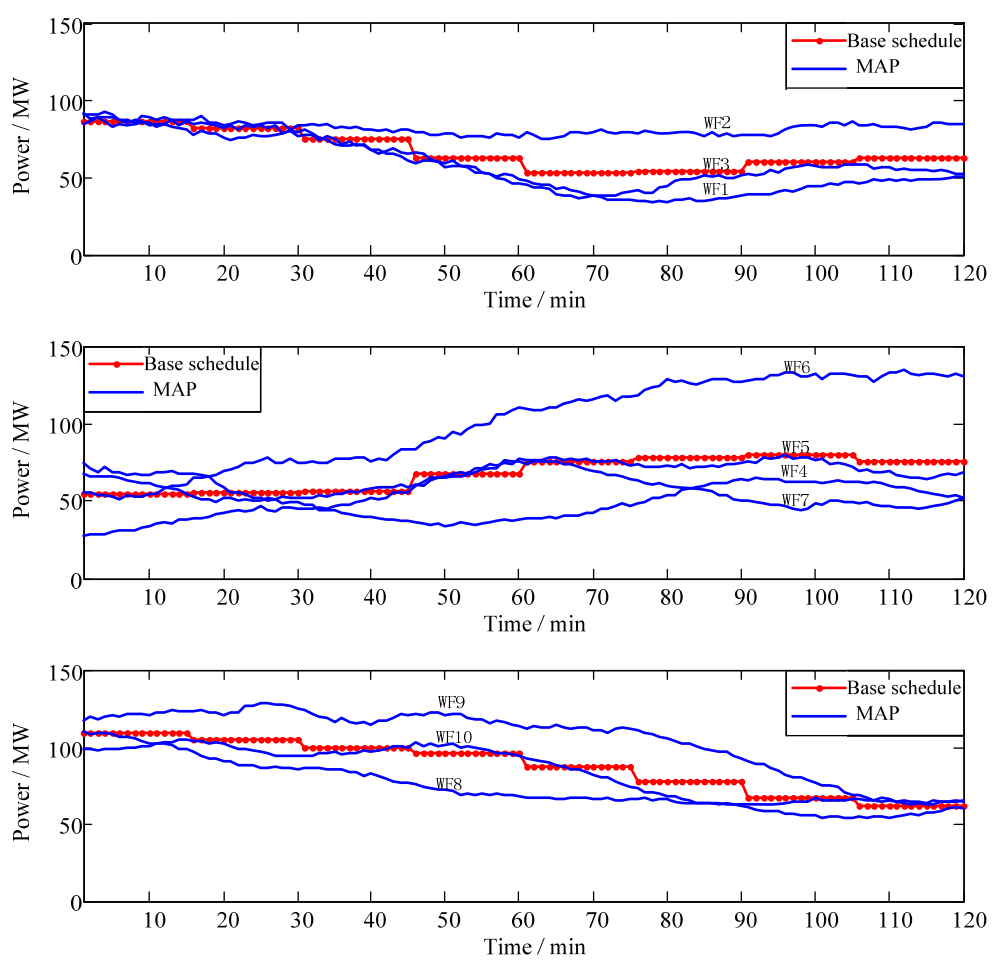

Figure 3 Base schedule and maximum available power (MAP) of wind farms.

The WFC base schedule is the sum of the base schedules of all wind farms in the WFC. The WFC regulation requirement is updated every 1 minute. The regulation quantity is calculated every 1 minute while the average regulation quantities, such as average ER quantity, average UR quantity and average OR quantity, are calculated every 15 minutes. The regulation revenue is calculated every 15 minutes based on the average regulation quantity. Since $N$ is equal to $8, C_{e}$ and $C_{p}$ are set to 5 times $C_{p s}$, which satisfies (31) and (32) to make the ER strategy as the Nash equilibrium. The unit revenue of generation $C_{g}$ is set as $27.75 \$ / M W h[31]$. 
$C_{e}, C_{p}$ and $C_{p s}$ are set according to Nash equilibrium condition which satisfies the (31) and (32). The WFC regulation requirement is treated as a new type of service. The regulation price from the PJM market is used which is $7.80 \$ / M W h$ [31]. If the ER strategy is adopted, the regulation revenue function $E_{r}$ is equal to $C_{e} \Delta P_{W F_{-}}^{i}+C_{p s} P_{W F}^{i}$ and $\Delta P^{i}{ }_{W F_{-} e}$ is equal to $P_{W F}^{i}$. The sum of $C_{e}$ and $C_{p s}$ is equal to $7.80 \$ / \mathrm{MWh}$. Because $C_{e}$ is set to 5 times $C_{p s}, C_{p s}$ is equal to $1.30 \$ / \mathrm{MWh}$. Then both $C_{e}$ and $C_{p}$ are $6.5 \$ / \mathrm{MWh}$.

\subsection{Comparison of WFC Control Performance}

The WFC control errors of the three control modes are shown in Figure 4. The average control error of Mode1, Mode2 and Mode3 are 70.53 MW, 7.58 MW and 2.89 MW, respectively. From the perspective of reducing the control errors, Mode3 is better than Mode1 and Mode2.

Only the base schedule of wind farms is tracked in Mode1. When the base schedule is less than the maximum available power, the power output of wind farm is limited to the base schedule and cannot be increased to reduce the WFC control errors. Therefore, the control errors of Mode1 are much larger than that of Mode2 and Mode3.

In Mode2, the WFC regulation requirement is equally distributed to all the wind farms. When the WFC control errors appear, wind farms regulate their power outputs to respond to the WFC regulation requirement. The WFC control error is reduced. However, wind farms' regulation ranges are proportional to the WFC regulation requirement. Such regulation ranges limit the regulation performance of wind farms from further decreasing the WFC control errors. .

In Mode3, wind farms compete to meet the regulation requirement and the regulation of wind farms is determined by the Nash equilibrium. The total regulation of wind farms is the WFC regulation requirement. As such, the WFC control errors are further reduced.

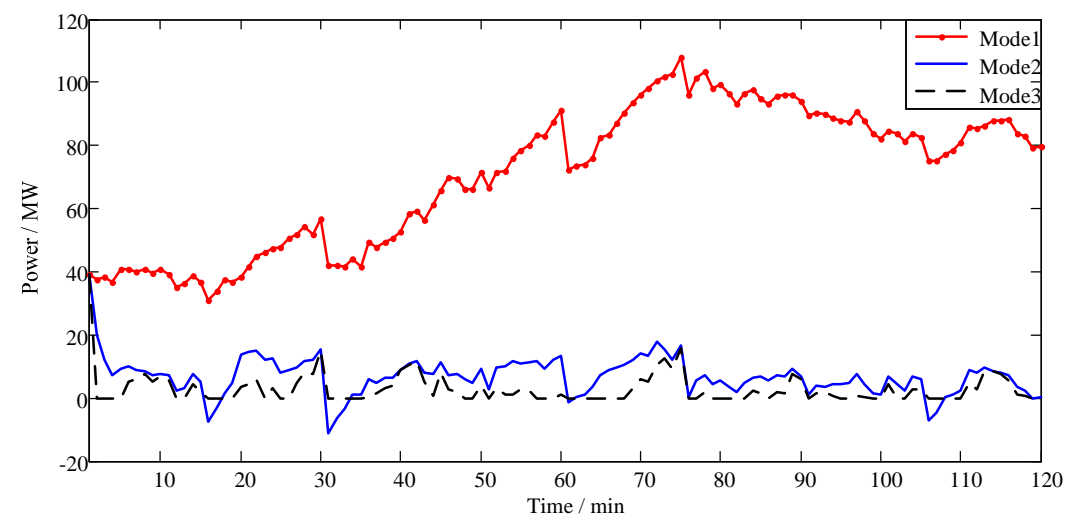

Figure 4 Control errors of the WFC.

Due to the dominant roles of WF2, WF6 and WF9 in meeting the WFC regulation requirement, the real-time power outputs and base schedules of WF2, WF6 and WF9 are shown in Figure 5 to illustrate the impact of control modes on the regulation delivered.

In Mode1, the control performance of WF2, WF6 and WF9 is limited. The real-time power output is limited to the base schedule of wind farms. The three wind farms cannot increase their power outputs to reduce the WFC control error.

In Mode2, WF2, WF6 and WF9 can increase their power outputs to track the WFC regulation requirement. Correspondingly, the real-time power outputs are larger than the base schedules of wind farms. However, the regulation gaps still exist, which limits the control performance of Mode2.

In Mode3, the WFC regulation requirement is not equally distributed to each wind farm. A larger regulation is achieved in Mode3 and it is equal to the WFC regulation requirement. Wind farms, such as WF6, can continue to increase their power outputs to reduce the WFC control errors. As such, in some time intervals, the real time power output of WF6 in Mode3 is larger than that of Mode2 as shown in Figure 5. Therefore, the control errors of Mode3 are smaller than that of Mode2. 

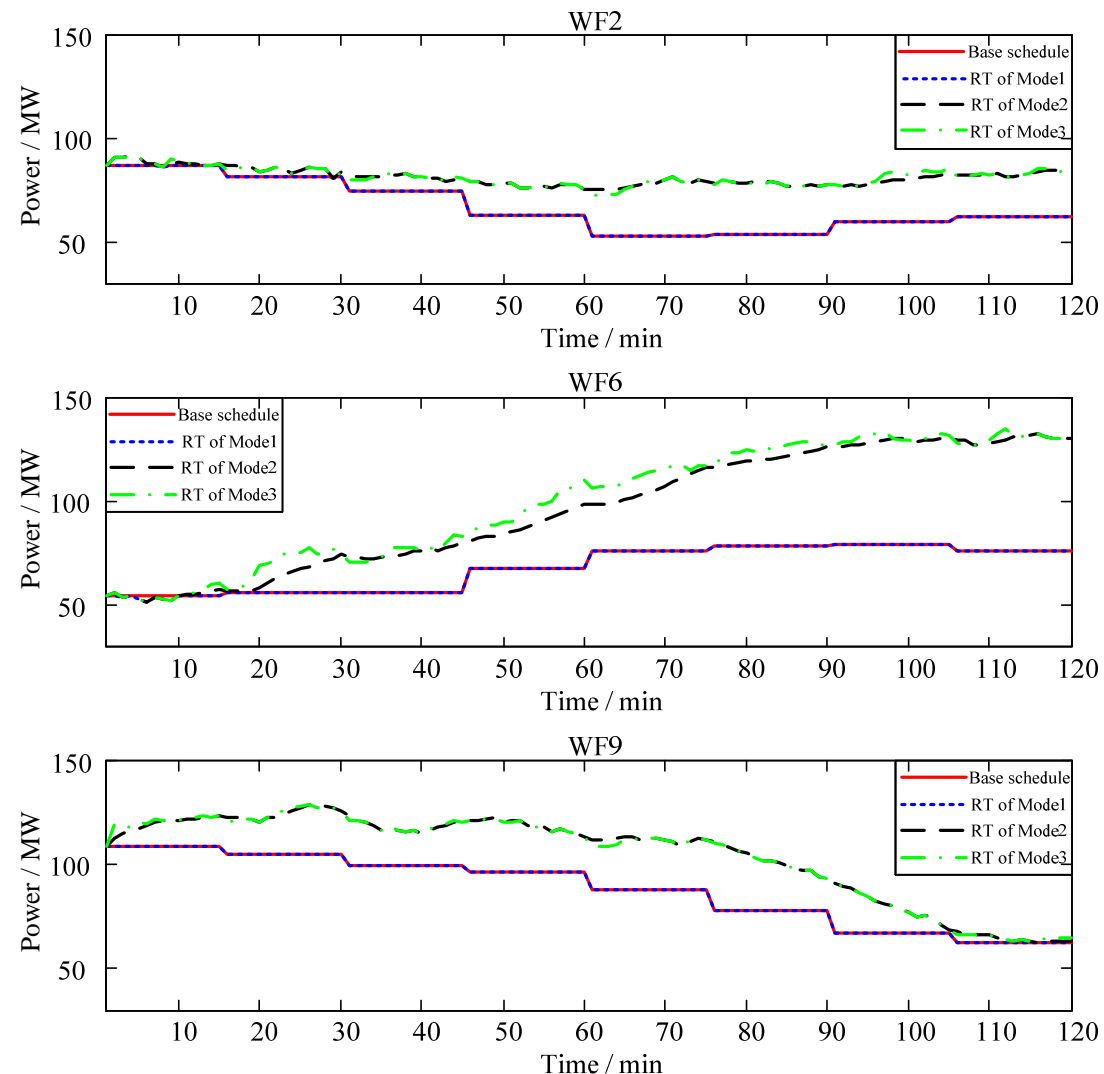

Figure 5 Real-time power outputs (RT) and base schedules of WF2, WF6 and WF9.

\subsection{Effectiveness of the Nash Equilibrium}

The game strategies of WF2, WF6 and WF9 are analyzed to evaluate the effectiveness of the Nash equilibrium. In the following analysis, except WF2, WF6 and WF9, other wind farms choose the ER strategy. Table 2 shows the chosen game strategies of WF2, WF6 and WF9. Mode3_i means the ith game strategy set in Mode3. 1 means the ER strategy and 0 means the maximum generation strategy which is a combination of UR and OR strategy.

Table 2 Portfolio of game strategies of wind farms.

\begin{tabular}{|l|l|l|l|}
\hline Mode & WF2 & WF6 & WF9 \\
\hline Mode3_1 & 1 & 1 & 1 \\
\hline Mode3_2 & 0 & 1 & 1 \\
\hline Mode3_3 & 1 & 0 & 1 \\
\hline Mode3_4 & 1 & 1 & 0 \\
\hline Mode3_5 & 0 & 0 & 1 \\
\hline Mode3_6 & 0 & 1 & 0 \\
\hline Mode3_7 & 1 & 0 & 0 \\
\hline Mode3_8 & 0 & 0 & 0 \\
\hline
\end{tabular}

The regulation revenue and control errors are listed in Table 3. The average WFC control errors are very small and are between 2.89 to $4.05 \mathrm{MW}$. A larger regulation revenue can be obtained with the ER strategy in a certain game strategy set. Take Mode3_1 and Mode3_3 as an example. When WF2 and WF9 choose the ER strategies, WF6 can get higher regulation revenue with the ER strategy, \$95.35, than with the maximum generation strategy, \$90.69. Similarly, for Mode3_2 and Mode3_5, when WF2 
chooses the maximum power strategy and WF9 chooses the ER strategy, larger regulation revenue can be obtained with the ER strategy for WF6. From Table 3, it can be seen that wind farms can get larger regulation revenue with the ER strategy than with the maximum generation strategy when the other wind farms have chosen certain game strategies.

Table 3 Regulation revenue and control error (CE)

\begin{tabular}{|l|l|l|l|l|l|}
\hline Mode & WF2/\$ & WF6/\$ & WF9/\$ & CE/MW & Overall cost/\$ \\
\hline Mode3_1 & 44.34 & 95.35 & 48.52 & 2.8949 & 188.21 \\
\hline Mode3_2 & 42.42 & 96.56 & 49.55 & 2.8949 & 188.53 \\
\hline Mode3_3 & 45.28 & 90.69 & 49.23 & 2.8949 & 185.20 \\
\hline Mode3_4 & 45.28 & 96.26 & 47.05 & 2.8949 & 188.59 \\
\hline Mode3_5 & 42.26 & 90.71 & 52.68 & 2.9119 & 185.65 \\
\hline Mode3_6 & 42.55 & 99.70 & 47.27 & 2.8949 & 189.52 \\
\hline Mode3_7 & 48.05 & 91.34 & 47.34 & 2.9076 & 186.73 \\
\hline Mode3_8 & 40.85 & 87.28 & 46.20 & 4.0450 & 174.33 \\
\hline
\end{tabular}

The total revenue of all the wind farms is the overall cost of the WFC shown in Table 3. The desired control mode (Mode3_1) has a relatively high cost. The total cost varies very little with different control modes except Mode3_8. In order to get a smaller control error, the total cost is worth being paid.

As a rational game player, a wind farm will choose the ER strategy. Wind farms will operate under Mode3_1 which is the desired control mode. As such, the proposed strategy can make wind farms respond to the WFC regulation requirement with the ER strategy.

\subsection{Regulation Quantity with Different Game Strategy}

Mode3_1 and Mode3_3 are selected to show the regulation quantities with different game strategies, which leads to different regulation revenue in Table 3.

For Mode3_1, all the wind farms choose the ER strategies to respond to the WFC regulation requirement. The average ER quantity, OR quantity and UR quantity of Mode3_1 and Mode3_3 are shown in Figure 6, Figure 7 and Figure 8, respectively.
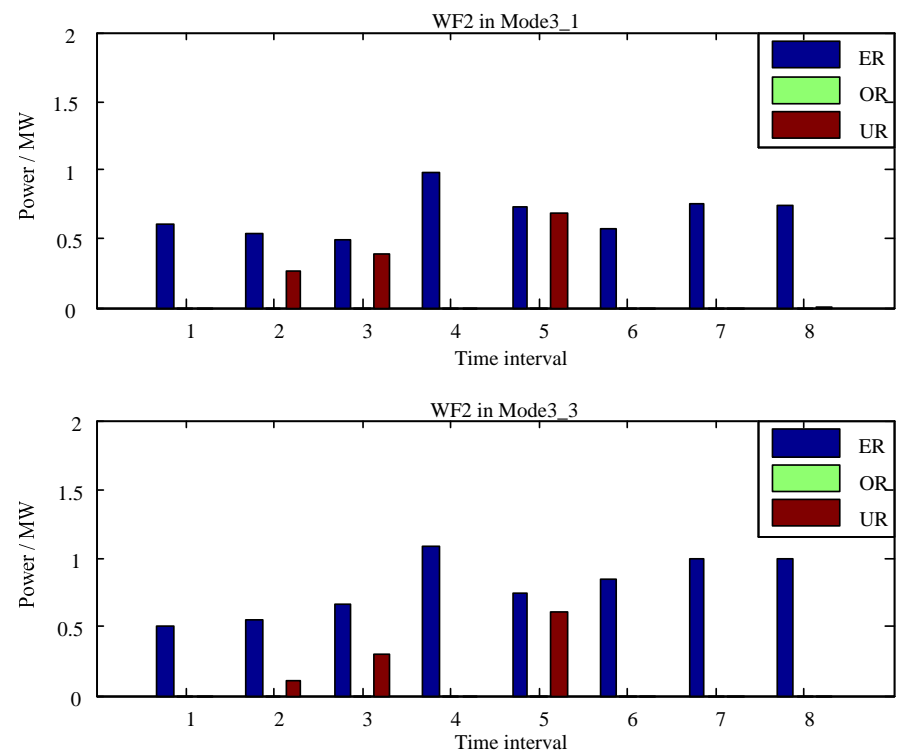

Figure 6 Average regulation quantity of WF2. 

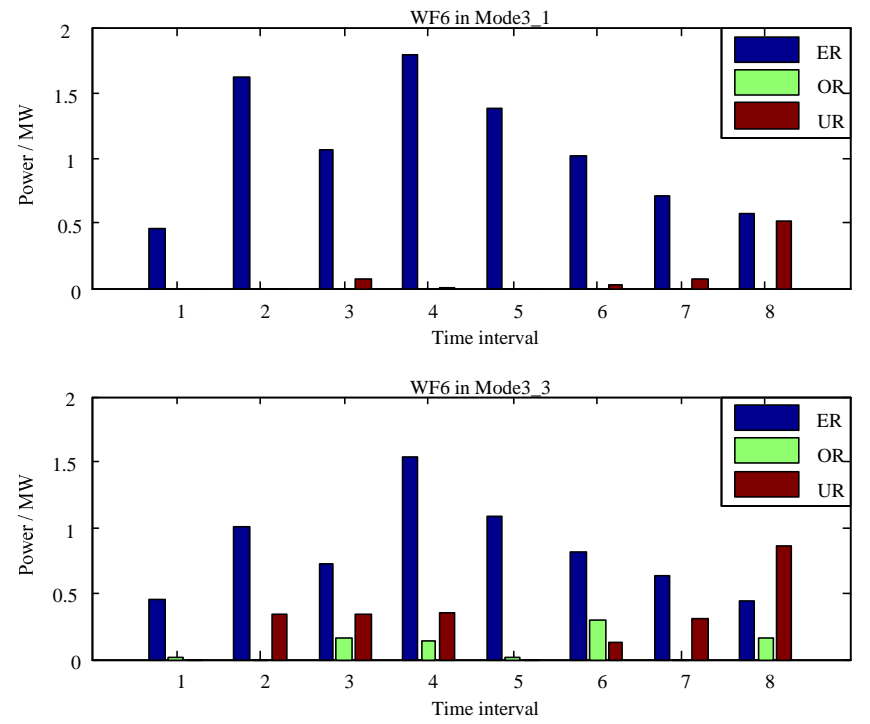

Figure 7 Average regulation quantity of WF6.
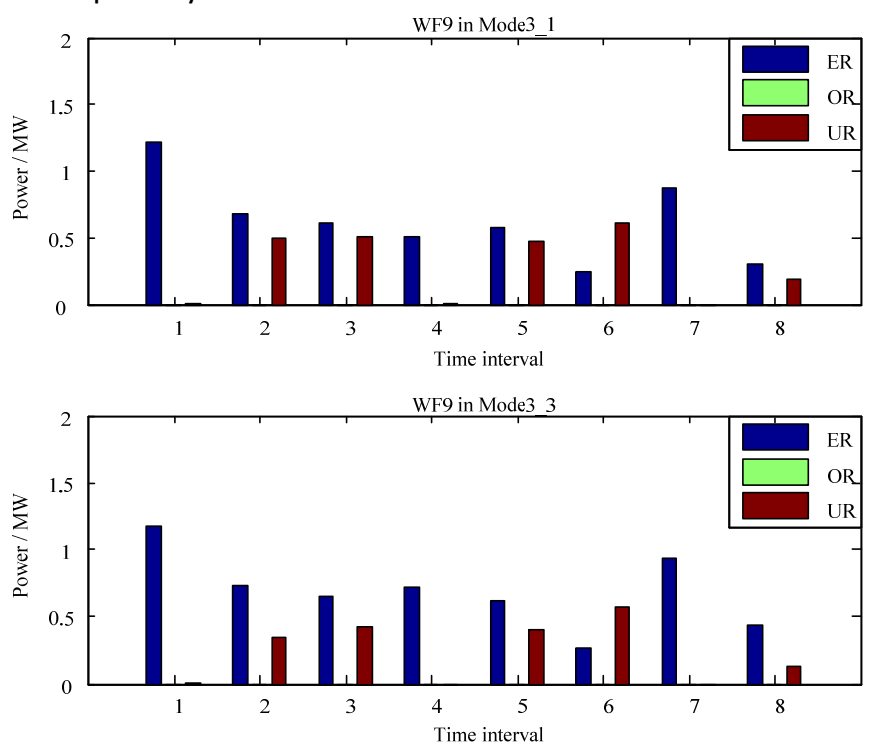

Figure 8 Average regulation quantity of WF9.

For Mode3_1, the ER quantity accounts for a large proportion of the regulation. Though the ER strategy is adopted, the UR quantity still accounts for a small proportion. During the down regulation, a down regulation minimum threshold $\Delta P_{\text {WF_min_th }}^{i}$ has to be satisfied. As the power support regulation quantity $P_{W F j}^{i}$ of some wind farms is relatively large, the down regulation minimum threshold is high. Considering the regulation speed limit, the down regulation minimum threshold cannot be satisfied and the UR quantity appears in Figure 6, Figure 7 and Figure 8.

For Mode3_3, WF6 chooses the maximum generation strategy while the other wind farms choose the ER strategies. The frequencies and values of the UR for WF6 increase as shown in Figure 7. Furthermore, the OR quantity of WF6 appears for Mode3_3 while there is no OR quantity for Mode3_1. For WF6, the OR and UR quantities of Mode3_3 is larger than that of Mode3_1, which leads to a larger punishment. Therefore, the regulation revenue of WF6 is reduced.

When WF6 increases its power output, the power outputs of WF2, WF9 and other wind farms decrease to reduce the WFC control errors. Therefore, the down regulation minimum thresholds $\Delta P_{\text {WF min_th }}^{i}$ of WF2 and WF9 decrease, which leads to smaller UR quantity as shown in Figure 6 and Figure 8. Furthermore, as WF2 and WF9 choose the ER strategies, the ER quantity increases. In the end, the regulation revenue of WF2 and WF9 increases. As a rational game player, WF6 chooses the ER 
strategy instead of the maximum generation strategy, which further verifies the effectiveness of the proposed strategy.

\section{Conclusions}

A non-cooperative coordination strategy based on the game theory is proposed to efficiently distribute the WFC regulation requirement during ramping events. Wind farms in the WFC compete to provide regulation to the WFC and the regulation from wind farms is coordinated by a non-cooperative game. A regulation revenue function is proposed to evaluate the competition process and the contribution of wind farms. The multi-time-interval Nash equilibrium condition of the non-cooperative game is derived. Then a simplified Nash equilibrium condition is proposed for the parameter setting of the regulation revenue function.

With the proposed non-cooperative coordination strategy, the WFC control error is further reduced compared to the existing control modes. When parameters of the regulation revenue function satisfy the multi-time-interval Nash equilibrium condition, wind farms with the ER strategy can get higher revenue than with combination of ER, OR and UR strategies. As rational participants, wind farms choose the ER strategy to provide regulation to the WFC during ramping events. Furthermore, the Nash equilibrium of the non-cooperative game makes wind farms choose the ER strategy to respond to the WFC regulation requirement and get more revenue.

\section{Acknowledgement}

The authors thank the National Basic Research Program of China (973 Program) (2012CB215101) and National Natural Science Foundation of China (No. 51177092) for the financial support to the research.

\section{References}

[1] Chang-Chien L, Sun C, Yeh Y. Modeling of wind farm participation in AGC. IEEE Transactions on Power Systems 2014; 29:1204-1211.

[2] Baeyens E, Bitar E Y, Khargonekar P P, Poolla K. Coalitional aggregation of wind power. IEEE Transactions on Power Systems 2013; 28: 3774-3784.

[3] Sevlian R,Rajagopal R. Detection and statistics of wind power ramps. IEEE Transactions on Power Systems 2013; 28: 3610-3620.

[4] Ganger D, Zhang J, Vittal V. Statistical characterization of wind power ramps via extreme value analysis. IEEE Transactions on Power Systems 2014; 29:3118-3119.

[5] Makarov Y V, Loutan C, Ma J, Mello P. Operational impacts of wind generation on california power systems. IEEE Transactions on Power Systems 2009; 24:1039-1050.

[6] Cui M, Ke D, Sun Y, Gan D, Zhang J, Hodge B. Wind power ramp event forecasting using a stochastic scenario generation method. IEEE Transactions on Sustainable Energy 2015; 6:422433.

[7] Kou B, Bai Y, Li L. A novel wind power generator system with automatic maximum power tracking capability. IEEE Transactions on Energy Conversion 2013; 28: 632-642.

[8] Gomis-Bellmunt O, Junyent-Ferre A, Sumper A, Bergas-Jane J. Control of a wind farm based on synchronous generators with a central HVDC-VSC converter. IEEE Transactions on Power Systems 2011; 26: 1632-1640.

[9] Hargreaves J J, Hobbs B F. Commitment and dispatch with uncertain wind generation by dynamic programming. IEEE Transactions on Sustainable Energy 2012; 3: 724-734.

[10] Khodayar M E, Shahidehpour M. Stochastic price-based coordination of intrahour wind energy and storage in a generation company. IEEE Transactions on Sustainable Energy2013; 4: 554-562.

[11] Makarov Y V, Etingov P V, Ma J, Huang Z, Subbarao K. Incorporating uncertainty of wind power generation forecast into power system operation, dispatch, and unit commitment procedures. 
IEEE Transactions on Sustainable Energy 2011; 2: 433-442.

[12] Ruttledge L, Miller N W, O'Sullivan J, Flynn D. Frequency response of power systems with variable speed wind turbines. IEEE Transactions on Sustainable Energy 2012; 3: 683-691.

[13] Delille G, Francois B, Malarange G. Dynamic frequency control support by energy storage to reduce the impact of wind and solar generation on isolated power system's inertia IEEE Transactions on Sustainable Energy 2012; 3:931-939.

[14] Miao Z, Fan L, Osborn D, Yuvarajan S. Control of DFIG-based wind generation to improve interarea oscillation damping. IEEE Transactions on Energy Conversion 2009; 24: 415-422.

[15] Kusiak A, Verma A. Monitoring wind farms with performance curves. IEEE Transactions on Sustainable Energy 2013; 4: 192-199.

[16] Rodriguez-Amenedo J L, Arnalte S, Burgos J C. Automatic generation control of a wind farm with variable speed wind turbines. IEEE Transactions on Energy Conversion 2002; 17: 279-284.

[17] Hansen A D, Sorensen P, lov F, Blaabjerg F. Centralised power control of wind farm with doubly fed induction generators. Renewable Energy 2006; 31: 935-951.

[18] Stamtsis G C, Erlich I. Use of cooperative game theory in power system fixed-cost allocation. IEE Proceedings - Generation, Transmission and Distribution 2004; 151: 401-406.

[19] Zolezzi J M, Rudnick $H$. Transmission cost allocation by cooperative games and coalition formation. IEEE Transactions on Power Systems 2002; 17: 1008-1015.

[20] Tsukamoto $Y$, Iyoda I. Allocation of fixed transmission cost to wheeling transactions by cooperative game theory. IEEE Transactions on Power Systems 1996; 11: 620-629.

[21] Ferrero R W, Shahidehpour S M, Ramesh V C. Transaction analysis in deregulated power systems using game theory. IEEE Transactions on Power Systems 1997; 12: 1340-1347.

[22] Mei S, Wang Y, Liu F, Zhang X, Sun Z. Game approaches for hybrid power system planning," IEEE Transactions on Sustainable Energy 2012; 3: 506-517.

[23] Mei S, Zhang D, Wang Y, Liu F, Wei W. Robust optimization of static reserve planning with largescale integration of wind power: a game theoretic approach. IEEE Transactions on Sustainable Energy 2014; 5: 535-545.

[24] Kazempour S, Zareipour H. Equilibria in an oligopolistic market with wind power production. IEEE Transactions on Power Systems 2014; 29: 686-697.

[25] Zugno M, Morales J, Pinson P, Madsen H. Pool strategy of a price-maker wind power producer. IEEE Transactions on Power Systems 2013; 28: 3440-3450.

[26] Baringo L, Conejo A. Strategic offering for a wind power producer. IEEE Transactions on Power Systems 2013; 28: 4645-4654.

[27] Ma Z, Callaway D S, Hiskens I A. Decentralized charging control of large populations of plug-in electric vehicles. IEEE Transactions on Power Systems 2013; 21: 67-78.

[28] Soliman H M, Leon-Garcia A. Game-theoretic demand-side management with storage Devices for the future smart grid. IEEE Transactions on Smart Grid 2014; 5: 1475-1485.

[29] Xu Q, Zhang N, Kang C, Xia Q, He D, Liu C, Huang Y, Cheng L, Bai J. A game theoretical pricing mechanism for multi-area spinning reserve trading considering wind power uncertainty. IEEE Transactions on Power Systems 2015; 31: 1084-1095.

[30] Qi Y, Liu Y. Wind power ramping control using competitive game. IEEE Transactions on Sustainable Energy 2016; 7: 1516-1524.

[31] PJM energy market, http://www.pjm.com/markets-and-operations/energy/real-time/Imp.aspx. 